\title{
LA ENFERMERÍA ESCOLAR: UN RECURSO NECESARIO PARA LA COMUNIDAD EDUCATIVA
}

\author{
Remedios Álvarez Terán \\ Profesora de FP Sanitaria en el IES Batalla de Clavijo \\ Maider Eguilaz Fanlo \\ Enfermera del Sistema Público Vasco de Salud \\ Saioa Miguel Moreno \\ Enfermera del Sistema Público Navarro de Salud
}

RESUMEN: A partir de la investigación llevada a cabo en cinco colegios riojanos con presencia de enfermería, se muestra la pertinencia de su incorporación en el entorno escolar. La ley de 1981 regula esta figura profesional en los centros de Educación Especial desde esa fecha, sin embargo, no termina de implantarse en los colegios de Educación Infantil, Primaria y Secundaria. El nivel de satisfacción respecto a las intervenciones llevadas a cabo en los centros educativos es elevado en los equipos directivos y muy elevado entre las familias. La colaboración entre los docentes, familias y enfermeras permite la adquisición de competencias en salud en el alumnado e incentiva la confianza y seguridad de las familias en la escuela como entorno promotor de salud además de contribuir a la mejora de la conciliación familiar en procesos crónicos y/o en urgencias.

PALABRAS CLAVE: Educación para la salud, enfermería escolar, conciliación familiar.

\section{SCHOOL NURSING: A NECESSARY RESOURCE FOR THE EDUCATIONAL COMMUNITY}

ABSTRACT: Based on the research carried out in five schools in La Rioja with a nursing presence, the relevance of their incorporation in the school environment is shown. The 1981 law regulates this professional figure in the Special Education centers since that date, however, it does not end up being implanted in the Primary, Secondary and Secondary Schools. The level of satisfaction with the interventions carried out in the schools is high in the management teams and very high among the families. Collaboration among teachers, families and nurses 
allows the acquisition of competencies in health in the students and encourages the confidence and security of families in the school as a health promoting environment as well as contributing to the improvement of family reconciliation in chronic processes and/or in The emergency room.

KEYWORDS: Education for health, school nursing, family reconciliation.

Recibido: 18/07/2017

Aceptado: 30/10/2017

Correspondencia: Remedios Álvarez Terán, IES Batalla de Clavijo, C/ Gral. Urrutia, 4, 26005 Logroño, La Rioja. Email: remediosat26@gmail.com.

\section{INTRODUCCIÓN}

La Organización Mundial de la Salud habla por primera vez de Educación para la Salud (EpS) en 1983, describiéndola como el conjunto de oportunidades para mejorar la alfabetización sanitaria de la población en relación con su bienestar y el aprendizaje de hábitos y habilidades personales que conduzcan a una mejor salud individual y de la comunidad (OMS, una definición que sigue hoy vigente.

La edad escolar se considera un periodo clave para desarrollar estrategias que permitan desarrollar competencias y habilidades para la vida.

La enfermería escolar nace con el propósito de preparar al alumnado para una vida independiente en sociedad y educarlo en los valores de la dignidad, la tolerancia y la igualdad, así como integrar a los y las menores que por inmadurez física y mental necesiten protección y cuidados especiales, siendo este un derecho humano de la infancia (CNDH, 2016).

Históricamente, el origen de la enfermería escolar se sitúa en Inglaterra en 1891 en el congreso londinense de Higiene y demografía, donde se describen los beneficios que aporta el personal de enfermería que acude a colegios de forma regular, primero a los privados y de educación especial y más tarde a los públicos (Drown, 1901).

Se empieza trabajando en los colegios de los barrios más necesitados, pero la tarea realizada es limitada por la escasez de los recursos. Sin embargo, la salud de esas poblaciones mejora con la iniciativa: se reduce el absentismo escolar y se generan nuevas conductas de salud que inciden en la comunidad educativa y familiar (Ossama, 2011). De ahí que, en 1897, analizando esos buenos resultados obtenidos con tan pocos medios se establece la primera fundación denominada The London SchoolNur-ses'Society (Sociedad de Enfermería Escolar Londinense). Eso contribuye a que se financien más puestos y su labor se expanda (Corral, 2016).

En 1909 Ilega a Boston (EEUU), donde se lleva a cabo un estudio en el que introducen a dos enfermeras en varios colegios para controlar el absentismo escolar debido a las enfermedades físicas y contagiosas. Es tal el éxito, que en 1926 la Organización Nacional de Enfermería en Salud Pública (ONESP) señala la eficacia 
en la educación sanitaria cuando interacciona el cuerpo docente con la enfermería, alcanzando un abordaje completo en la salud infantil y en la educación sanitaria a los padres.

En 1999 en Estados Unidos surge el término de "Schoolnursi". Actualmente, cuentan con 45.000 profesionales que dan cobertura a casi el 75\% de los centros. Según la American Federation of Teacher (AFT), existe un ratio de 1:1155. Estos resultados muestran como algunos gobiernos valoran la necesidad de disponer de un servicio de enfermería en las escuelas (Ossama, 2011).

En España, en 1886, Pedro de Alcántara García publica Tratado de Higiene Escolar. Ahí denuncia la ausencia de conductas higiénicas y alimenticias, critica los castigos escolares y, por primera vez, habla de la necesidad de crear 'botiquines' en dichos espacios. Él denuncia una situación insostenible en términos de salud, y habla de la necesidad de actuar (Alcántara, 1886).

Las primeras enfermerías escolares surgen en los Colegios Públicos de Educación Especial en la década de los ochenta al amparo de la Ley de Integración Social de los Minusválidos (LISMI), que en su artículo 27 considera que "(...) cuando la profundidad de la minusvalía lo haga imprescindible, la educación para minusválidos se llevará a cabo en Centros específicos". En tanto que el artículo 28 señala que cada centro "(...) deberá contar con el personal interdisciplinario técnicamente adecuado que, actuando como equipo multiprotesional, garantice las diversas atenciones que cada deficiente requiera" (Ley 13/1982, p. 11008).

La carta de Ottawa destaca la importancia de la colaboración intersectorial para el desarrollo de la EpS en las escuelas. En 1989, se firma un convenio de colaboración entre el Ministerio de Sanidad y el Ministerio de Educación, para instruir al profesorado en la Prevención de Enfermedades y en la Promoción de la salud. Sin embargo, los resultados obtenidos tras esta iniciativa legislativa han sido insignificantes. Los docentes se ven incapaces de desarrollar competencias que no les corresponde por su falta de conocimiento y de tiempo (Navarro, 2016).

Todas las leyes educativas que se suceden en esos años, y hasta el momento presente, mencionan la necesidad de generar hábitos saludables en el alumnado y de formarlo en conceptos básicos de salud. La última, la Ley Orgánica 8/2013, en su artículo 40 dice: "La enseñanza contribuirá a que el alumnado consiga los resultados de aprendizaje que le permitan trabajar en condiciones de seguridad y salud, así como prevenir los posibles riesgos derivados del trabajo" (Ley Orgánica 8/2013, p. 98887).

Además en La Rioja contamos con el impulso de la Consejería de Salud que, en colaboración con la Consejería de Educación, ejecuta diferentes programas de promoción de la salud: el Programa de Salud Bucocodental, para toda la población de Primaria y Secundaria, el Programa de Vacunaciones para el mismo colectivo, el de Promoción de la salud y educación en valores a través del cine, dirigido a dirigido a las familias del alumnado de $4^{\circ}$ de la ESO y Garantía social (Gobierno de La Rioja, 2017), el de Prevención del consumo de alcohol y otras drogas dirigido a Secundaria (Gobierno de La Rioja, 2017), el Programa de alimentación sana, y finalmente el Programa para la salud afectivo-sexual y educación, ambos en la educación Secundaria y Bachiller (Gobierno de La Rioja, 2017). 
Sin embargo y a pesar de todos los esfuerzos, el concepto de Educación para la Salud no se ha consolidado. La falta de continuidad en la formación de las y los más pequeños trae consigo consecuencias tan graves como el incremento de la obesidad infantil, mayor precocidad en consumo de tabaco, alcohol y drogas, aumento de enfermedades de transmisión sexual, embarazos no deseados, etcétera (Encinar, 2015).

Para intentar solventar estos y otros problemas, se plantea la incorporación en los centros educativos de la enfermería escolar, entendida como una figura que presta cuidados de salud a la comunidad educativa, con el objetivo principal de conseguir su máximo bienestar mental, físico y social pero que también educa e inculca hábitos de vida saludables de manera estable y no solo puntualmente dentro de un Programa.

Sin embargo en la actualidad, la enfermería escolar se encuentra en una situación de indefinición, ni está en todos los territorios del Estado ni en todos los tipos de centros. En el sector privado, la presencia de enfermería prestigia al centro, le aporta calidad. En algunos centros concertados, que generalmente dependen de órdenes religiosas, cuentan con personas voluntarias que se encuentran disponibles para ofrecer cuidados de salud, sin ser necesariamente profesionales. Y por último, en los centros públicos la enfermería está regulada en los centros de Educación Especial y ocasionalmente en todos aquellos que tienen alumnado con algún tipo de discapacidad o enfermedad crónica. La profesión no está presente en todos los casos y solo es a partir de 2001 cuando la enfermería escolar recibe un cierto impulso.

El objetivo de este estudio es presentar el estado real en La Comunidad Autónoma riojana, en los cinco centros que cuentan con profesionales de enfermería en sus plantillas.

\section{Método}

El presente estudio es un análisis cualitativo de un aspecto casi microscópico de la realidad, no obstante, aunque el grupo estudiado es muy pequeño, supone el total de esta colectividad. La información obtenida es examinada desde una perspectiva global, que responde a la interpretación subjetiva de las personas y de su ambiente, con todas sus complejidades intrínsecas y extrínsecas y siempre desde una perspectiva histórica. A través de este tipo de estudio se hace una descripción completa y detallada del tema en cuestión: la repercusión en la salud escolar de la presencia de enfermerías en cinco colegios riojanos.

En este apartado se hace referencia al marco teórico, las fuentes y la población diana.

\section{Marco teórico}

La enfermería escolar en nuestro contexto tiene un origen muy reciente como se señala en apartados anteriores. Esto, desde un punto de vista histórico, implica que nos referimos a una contemporaneidad muy presente. Por eso, la corriente historiográfica adecuada para este estudio es La Historia de Nuestro Tiempo. Un análisis que toma como eje el presente y que mantiene un diálogo permanente con otras ciencias sociales, el periodismo, la sociología, la literatura, etcétera. Esta 
perspectiva establece sus límites cronológicos en lo vivido, “... se trata de una idea elástica del presente, que se reelabora continuamente, pero que a su vez requiere de memoria" (Álvarez, 2007, p. 91).

De modo que nuestra investigación parte de la experiencia de las personas participantes, su visión como familias, como alumnado, como docentes acerca la teoría a la realidad.

\section{Fuentes}

Al tratarse la enfermería escolar de un campo profesional novedoso, existe escasa literatura científica en esta área. Esa es la mayor dificultad para investigar, sin embargo cuenta con la ventaja de las fuentes orales.

En el acceso a las fuentes, se han seguido dos técnicas documentales: por un lado, la lectura de textos (literatura científica, leyes, páginas web de los centros y de las Asociaciones de Enfermería), y por otro, las fuentes orales, a las que hemos accedido mediante entrevistas.

Tabla 1. Centros educativos estudiados, direcciones y profesionales de enfermería

\begin{tabular}{lll}
\hline \multicolumn{1}{c}{ Centro } & \multicolumn{1}{c}{ Dirección } & \multicolumn{1}{c}{$\begin{array}{c}\text { Profesional } \\
\text { enfermería }\end{array}$} \\
\hline CEIP General Espartero & Clara Martínez Montaña & E \\
CEIP Marqués de Vallejo & Pilar Arnedo Duarte & E1, E2, E3, E4 \\
CEIP Dr. Castroviejo & Roberto Estebas & E \\
CEIP Duquesa de La Victoria & Rosa Ezquerra Bañares & E \\
IES La Laboral & Roberto Castellanos Fonseca & E \\
Centro concertado Hermanos Maristas & Antonio Alegre Sánchez & E \\
\hline
\end{tabular}

Fuente: Elaboración propia.

Para iniciar la búsqueda documental, en primer lugar se han revisado diversas leyes de educación, salud, infancia y adolescencia. Con el fin de obtener la base teórica legislativa de la Enfermería Escolar: Ley de Ordenación General del Sistema Educativo, de 1990, Ley 1/1994 de Salud Escolar de la Comunidad Valenciana, Ley Orgánica de Educación (LOE) (2006), Ley Orgánica para la Mejora de la Calidad Educativa (LOMCE) (2013), y Ley de los Derechos de Salud del Niño y Adolescente de la Comunidad Valenciana (2008).

Para la literatura científica se ha recurrido a artículos extraídos de las siguientes bases de datos: PubMed, Scielo, Cuiden y Dialnet. Otra información procede de páginas web de organismos de salud de acceso público, ediciones web y revistas; así como de páginas específicas del ámbito de la Enfermería Escolar: Asociación Madrileña de Enfermería en Centros Educativos (AMECE), Sociedad Científica Española Enfermería Escolar (SCE3), Consejo de Enfermería de la Comunidad Valenciana (CECOVA), Asociación Americana de Maestros (AFT) y Organización Nacional de Enfermería en Salud Pública (ONESP). 
Respecto a las Fuentes orales, hemos contado con el testimonio de la comunidad educativa de los centros investigados: equipos directivos, familias (AMPAS) y enfermeras.

Las fuentes orales quedan así integradas en tres grupos humanos:

1. Ser miembro del equipo directivo de uno de los colegios que cumplen el requisito, preferentemente la directora o el director.

2. Ser madres, padres del alumnado matriculado en uno de estos centros.

3. Ser enfermera o enfermero en uno de los colegios seleccionados.

En un primer paso de la investigación, se concreta la relación de centros que cuentan con enfermerías. En segundo lugar, se envía un correo institucional a las direcciones de cada colegio explicando el objeto del trabajo y solicitando una entrevista. En tercer lugar, se conciertan las citas. En cuarto lugar, se realizan las entrevistas. Finalmente, contactamos con las AMPAS telefónicamente y por correo electrónico.

\section{Recogida y análisis de los datos}

Las entrevistas presenciales han durado entre una y dos horas. Todo el proceso se graba verbalmente y se transcribe. Posteriormente se agrupa y organiza la información por unidades de contenido estrechamente vinculadas a las preguntas realizadas.

Cuando citamos textualmente y con el fin de salvaguardar la confidencialidad de las personas entrevistadas, nos referimos a ellas/os con las siguientes siglas:

Ed: Entrevista individual director/a: Ed1, Ed2, Ed3, Ed4, Ed5.

Ef: Entrevista familia: Ef1 (Familias AMPA Maristas), Ef2 (Familias AMPA Duquesa de la Victoria).

Ee: Entrevista enfermera/o: Ee1, Ee2, Ee3, Ee4, Ee5, Ee6, Ee7, Ee8, Ee9.

\section{Población diana}

El propósito de este trabajo es conocer cómo se desarrolla la actividad en salud en los centros riojanos donde hay profesionales de enfermería trabajando. Estos son cinco en toda la región.

La edad de la población escolar de estos centros oscila entre tres y 22 años. Además, toda la comunidad educativa: familias, docentes, y otros profesionales pueden ser objeto de formación y cuidados por parte de las y los enfermeros.

En los centros de Educación Especial el alumnado tiene patologías psíquicas y/o cognitivas que le impide seguir una escolarización normalizada: distintos grados de discapacidad psíquica, plurideficiencias (físicas e intelectuales), déficits motores, alteraciones importantes de la conducta.

En los centros de Educación Infantil, Primaria y Secundaria, con alumnado entre los tres y los 16 años, puede haber personas con necesidades educativas especiales que en ocasiones requieren cuidados de enfermería: diabetes, asma y epilepsia son los trastornos estrella, pero también puede haber alergias muy diversas y otras patologías neurológicas que precisan de ayudas más específicas: sondajes vesicales, aspiraciones respiratorias, etcétera. Toda la población escolar puede requerir cuidados puntuales a demanda (epistaxis, gastroenteritis, golpes, heridas, etcétera). Finalmente, todo el colectivo es susceptible de ser educado para la salud. 
Los cinco colegios de la Comunidad Autónoma de La Rioja que cuentan con enfermerías son diferentes por su tipología, por su financiación y principalmente por el alumnado que acoge, aunque este siempre es individual y diferente a título personal.

Tabla 2. Centros educativos estudiados: financiación, características

\begin{tabular}{|c|c|c|c|}
\hline Centro & Financiación & Alumnado & Tipología \\
\hline General Espartero & Público & CEIP & Ordinario \\
\hline Marqués de Vallejo & Público & CEE & Especial \\
\hline Dr. Castroviejo & Público & CEIP & Ordinario \\
\hline Duquesa de La Victoria & Público & CEIP & Ordinario \\
\hline La Laboral & Público & IES & Ordinario \\
\hline $\begin{array}{l}\text { Centro concertado } \\
\text { Hermanos Maristas }\end{array}$ & Concertado & $\begin{array}{l}\text { Infantil, Primaria, } \\
\text { ESO, Bachiller }\end{array}$ & Ordinario \\
\hline
\end{tabular}

Fuente: Elaboración propia.

EI CEIP General Espartero, con una sola línea, tiene 225 plazas. Cuenta con consulta de fisioterapia y desde curso escolar 2016-2017, con enfermería.

El colegio Marqués de Vallejo está dedicado al Alumnado con Necesidades Educativas Especiales (ACNEE). Dispone de profesionales de enfermería desde 1981. En la actualidad tres, una persona por cada turno, ya que cuenta con internado.

EI CEIP Duquesa de la Victoria acoge a 700 niños y niñas en edades entre los tres y los 12 años. También dispone de personal de fisioterapia (compartido con otro centro) y enfermería en jornada completa.

EI IES La Laboral Lardero acoge a 1500 alumnas y alumnos e imparte enseñanzas en Educación Secundaria, Bachillerato y Formación profesional. Cuenta con Residencia, lo que generó desde su apertura en 1974 la necesidad de enfermería.

El centro concertado Maristas, situado en el extrarradio logroñés, acoge a 1800 escolares distribuidos en Educación Infantil (dos ciclos), Primaria, Secundaria y Bachiller. Tiene enfermería desde hace una década.

\section{Resultados}

En todos ellos, las y los enfermeros realizan sus funciones con toda normalidad como tendremos oportunidad de ver:

Entiendo este trabajo como una consulta externa un poco a demanda, tienes que hacer un campo de educación. Yo así me lo he planteado y así lo trabajo (Ee5).

Ha sido una bomba, en las encuestas de calidad que mandan a los familiares nos hemos disparado (Ee1).

Los espacios donde despliegan su actividad tienen un aspecto similar: ausencia de barreras arquitectónicas, ascensores, barandillas en las escaleras, rampas para acceder en silla de ruedas o con otros dispositivos, lavabos con suficiente espacio para ser uti- 
lizados por personas con movilidad reducida, iluminación natural, un patio de recreo parcialmente cubierto. El IES La Laboral cuenta con múltiples botiquines ubicados en distintos puntos del centro. En el Marqués de Vallejo tienen un comedor amplio clasificado en dos zonas: una para quienes requieren ayuda para comer, este es más amplio y contiene sillas especiales; y otra zona para quienes gozan de autonomía en esta tarea.

Como vemos, todos los centros comparten multitud de aspectos, y todos tienen algunas especificidades que son producto de la colaboración enfermera y equipos directivos en cada ocasión.

\section{Los equipos directivos opinan}

Las personas responsables de la gestión en cada centro manifiestan, en general, la tranquilidad y la calidad en términos de salud que supone contar con estas y estos profesionales. Si bien es cierto que la opinión no es compartida por la totalidad.

No querríamos por nada del mundo no tener enfermera. Tenerla es un lujo. Son todo ventajas, un plus. Participa muy activamente dentro del centro, lo principal es lo suyo, pero hace muchas cosas diferentes. Incluso a veces hay que poner límites, porque en otro caso, los niños abusarían de su trabajo. Sobre todo aquellos con necesidad de atención debido a que vienen de familias desestructuradas o con problemas económicos. Lo hemos estudiado y son los que más acuden a la enfermería (Ed4).

En un centro (...) vale la pena tener una persona en atención continua (...), es un plus que tiene el colegio, las familias se sienten más seguras. Aunque, donde hay enfermera hay más enfermos (Ed1).

Solo en un caso se muestra cierto escepticismo:

Hacemos uso de ella para atender las urgencias del instituto también, pero su plaza está por la residencia (...). Su consulta está allí, y su tarea principal se reduce a atender a demanda los problemas de los residentes (...). Si un residente tiene que ir al Centro de Salud ella es quien se encarga de acompañarles (Ed5).

No podríamos no contar con la figura de enfermería, es un centro de educación especial, su presencia es imprescindible (...) en las visitas con los padres se comenta la disposición de la enfermera y ellos por su parte lo exigen (Ed3).

Las referencias a la calidad en salud, prestigio social y seguridad para las familias con niñas y niños con necesidad de cuidados son otras de las aportaciones muy valoradas. La enfermería aparece publicitada en las páginas web y es uno de los espacios que las familias visitan con curiosidad en las jornadas de puertas abiertas cada año.

En jornada de puertas abiertas siempre está la enfermera presente. Ella cuenta a todos los padres que su trabajo prioritario es cuidar a los niños que tienen una enfermedad pero que a su vez atiende a demanda (...). Los padres la valoran mucho no, muchísimo (...) es muy importante para los niños también, psicológicamente hablando les ayuda mucho (...) contamos con proyectos de inteligencia emocional (...) la dirección la apoya en todo y el profesorado también. Ni las familias ni nosotros nos imaginamos el colegio sin enfermera (Ed4). 
Tuvimos la jornada de puertas abiertas y todos los padres estaban encantados (...) tanto a las familias como a nosotros nos da una seguridad, por nuestra parte la enfermera estaría siempre (...). La mamá de un niño diabético se puso a llorar cuando se enteró de que contaríamos con una enfermera este año, para ella supuso la tranquilidad absoluta ( $\operatorname{Ed} 2)$.

Comunicamos a las familias que hay enfermera (...). Cuando se enteran, se les pone cara de alivio y felicidad" (Ed5).

A mí como director me da seguridad, tienes a una profesional. Yo nunca he tenido enfermera en ningún otro colegio de los que he estado, entonces todo se limitaba a la persona que estaba en portería (...) sí que es verdad que te topas con muchos dolores indeterminados, es un reclamo en los recreos, sobre todo en niños con necesidades afectivas (...) aun así, lo veo como algo positivo y en lo que se debería invertir, aunque entiendo que si no ocurre es porque el ministerio no cuenta con los medios suficientes (Ed1).

"Lo ideal sería que en todos los centros hubiera una enfermera. A los padres les da seguridad, se van a trabajar sabiendo que sus hijos están bien atendidos, puede trabajar ambos en la familia (...).Hacen mucho caso y confían en el criterio de esta profesional (...), es contraproducente no tener enfermera. Como docentes no podemos dar ningún tipo de medicación, pero si no lo hacemos te denuncian por negligencia (Ed2).

En general, las enfermerías proporcionan seguridad, gestionan los recursos sanitarios y dan la respuesta profesional a las necesidades en salud en un mundo cada vez más complejo también en los entornos educativos.

\section{Percepción de las familias}

La totalidad de las familias preguntadas afirma que la presencia de enfermerías en los centros solo ofrece ventajas, tanto a nivel sanitario como social.

Creo que es muy positivo que exista un profesional que gestione las posibles necesidades de atención de los niños, que realice las atenciones pertinentes y que, si es necesario, nos avise (Gdf1).

Valoran sobre otros factores la seguridad que les proporciona. Aunque solo sea para una primera valoración:

Te da cierta tranquilidad. Aunque, por supuesto, hay que llamar a los servicios de emergencias o urgencias (si el problema lo requiere). Podemos hablar desde un dolor de cabeza hasta un ataque de epilepsia (Gdf1).

Creo que es bueno disponer de este servicio en el centro, toda vez, que puede dar una primera valoración de lo que le ocurre al alumno y derivarlo si fuera necesario al centro de salud, hospital, etcétera (Gdf2).

Agradecen y valoran que exista un profesional sanitario que gestione las pequeñas urgencias y preste los primeros auxilios:

Me gusta cómo reacciona en la actualidad. Cuando hay un problema de salud se avisa a la enfermera del centro y lo primero que hace es evaluar el estado del niño y a continuación te llama para darte su opinión profesional y decide con los padres el modo de actuar en cada caso (Gdf1). 
Considero imprescindible su presencia (Gdf2).

En las pocas ocasiones en que se ha necesitado ha sido muy correcta. Me han avisado y ha estado con mis hijos hasta que he llegado a recogerlos. Yo estoy más tranquila porque están atendidos. Por otra parte, no se interrumpen las actividades de los otros niños de clase por estar su profesor acompañando a mi hijo hasta que yo llegue (Gdf1).

También la mayoría cree que el personal enfermero debe contribuir a reciclar al profesorado y al personal del centro en la atención, asistencia y orientación de los problemas de salud de la población escolarizada. En todos los centros lo hacen en un grado u otro, imparten sesiones sobre salud bucodental, diabetes, primeros auxilios, responden a las demandas que detectan y colaboran con los asuntos para los que se solicita su ayuda:

A parte de las urgencias que se puedan presentar, crear programas sanitarios para detectar problemas como la obesidad, buena educación sanitaria, prevención de enfermedades, actuación ante dicho riesgo. Todo ello merece la pena y es trabajado. Tener esta faceta cubierta por la enfermera repercute en evitar el absentismo escolar de los niños y laboral de los padres (Gdf1).

En todos los centros contribuyen, en diferente grado, a ejecutar los programas de la Consejería de Salud riojana:

Sí que se realizan actividades en el centro de manera continuada, pero creo que nunca es suficiente. Por supuesto, dirigidos por nuestra enfermera. A mí lo que más me preocupa es que se detecten de manera temprana signos y síntomas de enfermedades como la diabetes, la epilepsia o la obesidad infantil. Por eso creo que hay que incidir ahí (Gdf1).

Realizados por la enfermera de manera puntual. Creo que no son suficientes. Hablan sobre la higiene bucodental y la alimentación, lo cual está bien (Gdf2).

Es muy necesario que sea la enfermera quien lleve a cabo estos protocolos. Aunque los haga de manera puntual, siempre se puede mejorar. Supongo que como a todo padre lo que más me importa es que estén sanos (Gdf2).

Respecto a la educación para la salud, creen que debería asumir mayor responsabilidad ya que tienen mayor asertividad que las familias para inculcar estilos de vida saludables:

(...) y les recuerda que deben mantener unos hábitos saludables respecto a su alimentación, ejercicio, atención a los posibles peligros, etcétera (Gdf1).

Cuando se pregunta si conocen las funciones que desempeña enfermería, afirman estar bastante al tanto. Algunas familias creen que deberían asumir incluso mayores responsabilidades:

Lo considero necesario sí. Ya que creo que muchos padres piensan que solo están para curar las heridas que se hacen en el colegio y poco más. Reuniéndonos con ella podríamos abarcar muchos más temas importantes para la salud de nuestros hijos (Gdf1). 
La enfermera del centro, cuando hay un problema de salud, realiza esas "tutorías", desde el momento que te notifica las incidencias y sigue su evolución. Tutorías periódicas, no creo que sean necesarias ni tampoco posibles, teniendo en cuenta que hay una enfermera y en torno a 1000 niños (Gdf1).

Conocemos el papel que desempeña la enfermera en nuestro colegio, por eso creo que más que necesario ha de ser de fácil acceso para la persona que realmente lo necesite (Gdf2).

La enfermera debe de ser accesible si realmente hay un problema. No veo mal que destine un tiempo a atender a los padres. Pero con sentido común (Gdf1).

Cuando se detecta un problema es fundamental. Pero para ello es muy importante el trabajo de la prevención (Gdf1).

Las familias tienen la certeza de que, durante las horas escolares, sus hijas e hijos obtienen la máxima calidad en términos de salud. A ellas también les reporta posibilidades de mayor conciliación y tranquilidad.

Creo que son todo ventajas. La tranquilidad de tener un profesional de la salud en el colegio que incluso evita tener que salir del trabajo en determinados momentos y que les ayuda en muchos aspectos tanto de salud física como emocional. No veo desventajas (Gdf1).

Para mí son todo ventajas. La posible desventaja sería el coste de dicho servicio, pero creo que es un dinero bien empleado (Gdf1).

Me da mucha tranquilidad, si pasa algo sé que hay un profesional. Apoya a los niños en temas más allá de la propia salud física (Gdf2).

La tranquilidad de tener una profesional que sabes que va a atender a tus hijos ante una situación de emergencia o simplemente cuando no se encuentran en buen estado de salud (Gdf1).

En mi opinión es muy positivo. Es un apoyo que, si se tiene, es muy conveniente para los profesores. Sobre todo, porque hoy en día y con la tendencia a la integración se pueden dar muchas casuísticas que no son ámbito ni conocimiento de los profesores. Para los casos particulares de necesidades concretas, el personal sanitario es muy conveniente y para el resto de niños que no tengan problemas también, puesto que en el caso de que haya una urgencia, sus actividades no se ven interrumpidas ya que el profesor sigue con su actividad al tener otra persona que se ocupa de esa urgencia (Gdf2).

Como posible desventaja el coste que tiene, pero creo que cuando se ve y se conoce su trabajo se asume totalmente (Gdf1).

Ciertamente, y a pesar de contar con un amplio respaldo de toda la comunidad cuando ya está implantada la figura, al no estar regulada no todo el alumnado riojano puede beneficiarse de estos servicios. Como los recursos siempre son limitados, surgen otras necesidades más pujantes que van relegando su implantación a la demanda puntual por parte de los centros o de las familias. Así se viene Ilevando a cabo su incorporación en los últimos años. 


\section{Las y los enfermeros escolares se pronuncian}

Todas las y los enfermeros que han participado en este estudio Ilevan a cabo las funciones que regulan la profesión:

Incumbe a la profesión de enfermería la responsabilidad de proporcionar de forma individual o, en su caso, de forma coordinada (...), los cuidados propios de su competencia, al individuo, a la familia y a la comunidad... (Real Decreto 1231/2001, artículo 54, p. 24).

(...) Los profesionales sanitarios desarrollan, entre otras, funciones en los ámbitos asistencial, investigador, docente, de gestión clínica, de prevención y de información y educación sanitarias (Ley Orgánica de 44/2003, p. 5).

De modo que asisten, la que viene a decir que llevan a cabo actividades en salud según protocolos; hacen el seguimiento y control de quienes padecen enfermedades crónicas como diabetes, asma, enfermedades celíacas u otras afecciones; realizan los pertinentes planes de atención de enfermería al alumnado que así lo requiere y actúan ante emergencias o incidencias que surgen en el horario escolar:

La enfermería está en contacto con médicos, para que les proporcione temas de informe psiquiátricos o tratamientos (Ee7).

He pedido un psiquiatra de referencia, que en un momento puntual pueda ayudarte, que conozca a los escolares. Hay alumnado que acude al psiquiatra de adultos de Calahorra, y mayor coordinación facilitaría mucho el trabajo (Ee3).

Todo lo que acaece desde un punto de vista clínico se recoge en las Historias de Enfermería. Y se informa a las familias:

A primera hora atiendo siempre la demanda, dolores de cabeza, de estómago, alguna duda que tengan de algún tratamiento la resuelvo, etcétera. La demanda la atiendo igual que el resto (E7).

Esta es la función más ejecutada en horas de dedicación y también la más conocida socialmente.

Casi todas imparten charlas sobre aspectos diversos de la salud, si bien esperan desarrollar más este aspecto en el futuro. No siempre disponen de tiempo o de espacios generados con este fin. De este modo desarrollan la competencia docente:

Doy charlas en función de la necesidad, en el caso del niño diabético di una charla sobre la diabetes en clase, para normalizar la situación. Por sí, por ejemplo, vamos de excursión y ocurre una hipoglucemia, que no se asusten y entiendan lo que es la diabetes (Ee4).

La competencia administrativa ocupa mucho de su tiempo, al ser el nexo de unión entre los centros y las diferentes administraciones de salud, publicitan y gestionan todo el grueso de permisos familiares para revisiones bucodentales o vacunas, consultas, cancelaciones, resultados de las revisiones, nuevas citas, etcétera.

También dentro de esta competencia, generan la Historia de Enfermería. En primer lugar detectan entre el alumnado de nuevo ingreso aquel susceptible de requerir cuidados profesionales en el horario escolar: 
Los familiares nos proporcionan una Ficha de Salud al inicio del curso, y yo los registro en la base de datos del ordenador, y voy sumando ahí las actividades correspondientes que les realizo, y la medicación que les doy. Una enfermera si no registra no es nada (Ee1).

Tenemos un registro que se les da a los familiares del alumnado al inicio de la escolarización, es una Ficha de Salud, donde pone que, si tienen alguna medicación crónica, lo comuniquen (Ee2).

( ) nos dan un listado con el informe médico y yo hago el registro de sus Historias en la base datos del alumnado (Ee5).

También realizan los pedidos sanitarios, tanto del material fungible como del permanente (camillas, flexos, gasas, guantes medicamentos), y todo ello está inventariado:

( ) al principio del curso hacemos una lista de lo que creemos que vamos a utilizar. Sí después necesitamos algo más, en un momento puntual, podemos ir a la farmacia a comprarlo, pero me da envidia no poder tener el material del hospital, por ejemplo, una venda de crepé (Ee7).

Al inicio del curso me dan un presupuesto, yo lo gestiono, encargo lo necesario a la empresa o voy a la farmacia. Sí me paso de ese presupuesto no importa, me lo amplían sin problema. Eso sí, sería mucho más cómodo que nos faciliten el material desde el hospital (Ee2).

Finalmente, la competencia investigadora es la menos desarrollada. Ciertamente recogen los datos de manera científica, los analizan y sirven para proyectar el trabajo en foros, congresos o reuniones profesionales y para mejorar la práctica cotidiana e incrementar los conocimientos sobre salud escolar. También sirven para establecer vías de investigación.

En relación con otros aspectos formales, hay que decir que, en consonancia con su trayectoria profesional, casi en todos los casos visten con bata blanca, casi, porque en otras ocasiones llevan pijamas coloridos:

No hace falta llevar una bata blanca con jeringas, yo estoy más cómoda así, y esto también influye en la percepción que tienen hacía mi trabajo, se sienten menos calificados (Ee1).

Los recursos materiales de las consultas son similares: algodón, gasas estériles, tiritas, vendas, esparadrapo, suturas quirúrgicas, compresor, tijeras, pinzas, estetoscopios, esfingonómetros, glucómetro, termómetros, pulsioxímetro, linternas, muletas, mascarillas güedel, maletín de urgencias, silla de ruedas, bala de oxígeno y ambús.

La medicación disponible es muy básica en todos los centros: paracetamol, ibuprofeno, aspirina, antiácidos, omeprazol, antihistamínicos, adrenalina, cremas para las picaduras, etcétera, solo se dispensa por orden médica. Y luego los medicamentos específicos de cada tratamiento particular.

Casi siempre tienen autonomía para decidir cómo y en qué se utiliza el presupuesto con el que cuentan. 


\section{Discusión}

La enfermería escolar desarrolla cuatro funciones básicas: asistencial, docente, gestora e investigadora. Aunque la docente puede ser la más rentable a largo plazo, solo se visibiliza la asistencial, que desde luego es fundamental y actualmente casi la única razón para que exista esta figura en los centros.

En los colegios estudiados, el personal enfermero desarrolla sus funciones con autonomía, aunque no todas las personas entrevistas comparten este punto de vista, ya que dicen sentir la presión del colectivo mayoritario con el que conviven, el personal docente. En algunas ocasiones no resulta fácil comprender las respectivas necesidades profesionales ni compartir parcelas de conocimiento y poder. También las familias ejercen su presión, exigiendo atenciones y cuidados que no son de su competencia, por ejemplo, la toma de partido en asuntos familiares o la prescripción o modificación de ciertos tratamientos médicos.

En general, toda la comunidad está satisfecha con la presencia de enfermeras o enfermeros: las familias, los equipos directivos y las y los docentes. Para las primeras, contribuye a favorecer la conciliación de la vida familiar y laboral, y solventa las consultas con respecto a la salud de sus criaturas. En relación con los segundos, da calidad y prestigio al centro que dispone de estas profesionales. El colectivo docente se ve liberado de ejercer responsabilidades que no son de su competencia: diagnosticar y o cuidar mientras llegan las familias a hacerse cargo de sus retoños.

Todas las profesionales entrevistadas muestran un grado alto de satisfacción, se sienten reconocidas y realizadas. A corto plazo, no ven la necesidad de cambiar de puesto. Como vemos todos los centros comparten multitud de aspectos y todos tienen algunas especificidades que son producto de la iniciativa enfermera y de la colaboración o no de algunos directivos en cada ocasión.

Consideramos que este trabajo resulta muy valioso por su novedad, es el primero realizado en La Rioja, aunque que también pensamos que es necesario seguir investigando, divulgando y dando su lugar a esta variable de la profesión enfermera que, aunque desconocida o poco visible, no es menos relevante y contribuye a mejorar la calidad de vida de la comunidad escolar y, por lo tanto, de toda la comunidad.

\section{REFERENCIAS BIBLIOGRÁFICAS}

Alcántara García, Pedro de (1886). Tratado de higiene escolar: guía teórico-práctica para uso de los inspectores, maestros, juntas, arquitectos. Alicante: Biblioteca Virtual Miguel de Cervantes. Recuperado de http://www.cervantesvirtual.com/ obra-visor/tratado-de-higiene-escolar-guia-teoricopractica--0/html/fefc21 f0-82b111df-acc7-002185ce6064_24.html\#I_1_.

Alcántara García, Pedro de (1900). Teoría y práctica de la educación y la enseñanza: curso completo y enciclopédico de Pedagogía. Madrid: Librería de Hernando y Compañía.

Álvarez Terán, R. (2008). "Historia Contemporánea, historia de nuestro tiempo e historia de género: estado de la cuestión". En C. Navajas Zubeldia y D. Iturriaga Barco (Coords.), Crisis, dictaduras, democracias: I Congreso Internacional de 
Historia de Nuestro Tiempo (pp. 91-103). Logroño: Universidad de la Rioja. Recuperado de https://dialnet.unirioja.es/servlet/articulo?codigo=2676327.

Comisión Nacional de los Derechos Humanos (2014). Derechos humanos de niñas, niños-adolescentes. México: CNDH. Recuperado de http://www.cndh.org.mx/ Ninos_Derechos_Humanos.

Consejería de Salud, Dirección General de salud pública. Programa promoción de la salud. Recuperado de http://www.larioja.org/mujer/es/salud/programa-promocionsalud.

Consejería de Salud, Dirección General de salud pública (2017, 11 de agosto). Salud promueve un programa de prevención del consumo de alcohol y otras drogas dirigido a alumnos de secundaria de La Rioja. Recuperado de http://www.larioja. org/comunicacion/es/portavoz/salud-promueve-programa-prevencion-consumoalcohol-drogas-d.

Gobierno de La Rioja (2017, 12 de mayo). El Gobierno de La Rioja promueve programas de promoción y educación para la salud en el ámbito educativo. Recuperado de http://www.larioja.org/comunicacion/es/noticias/gobierno-rioja-promueve-programas-promocion-educacion-salud.

Corral Rodríguez, O. (2016). La Enfermería Escolar: Rol, funciones, y efectividad como promotora de la salud. V (pp. 1-21). Santander: Escuela Universitaria de Cantabria. Recuperado de https://repositorio.unican.es/xmlui/bitstream/handle/10902/8918/ Corral\%20Rodriguez\%200.pdf? sequence $=1$.

Drown, L. L. (1901). The London Public-School Nursing. The American Journal of Nursing, 1(4), 270.

Encinar Casado, A. (2015). La situación de hoy en día. Revista Enfermería C y L. Enfermería Escolar. Recuperado de http://www.revistaenfermeriacyl.com/index.php/ revistaenfermeriacyl/article/view/144.

González García, N. y López langa, N. (2012). Antecedentes históricos y perfil de la enfermera escolar. Metas de enfermería, 15(7), 50-54. Recuperado de https:// dialnet.unirioja.es/servlet/articulo?codigo $=4128451$.

Ley 13/1982, de 7 de abril, de integración social de los minusválidos. BOE $\mathrm{n}^{\circ} 103$, de 30 de abril de 1982 .

Ley Orgánica 1/1990, de 3 de octubre, de Ordenación General del Sistema Educativo. BOE no 238, de 4 de octubre de 1990.

Ley Orgánica de 44/2003, del 23 de noviembre. Ordenación de los Profesionales Sanitarios. BOE n ${ }^{\circ} 280$, de 22 de noviembre de 2003.

Ley Orgánica de Educación 2/2006, de 3 de mayo, de Educación. BOE nº 106, de 4 de mayo de 2006.

Ley del 8/2008, de 20 de junio, de la Generalitat, de los Derechos de Salud de Niños y Adolescentes. BOE $\mathrm{n}^{\circ} 171$, de 16 de Julio de 2008.

Ley Orgánica 8/2013, de 9 de diciembre, para la mejora de la calidad educativa, BOE $\mathrm{n}^{\mathrm{o}} 295$, de 10 de diciembre de 2013.

Navarro Fernandino, M. (2016). Enfermería Escolar, una revisión bibliográfica (Trabajo fin de grado, Tortosa, Universitat Rovira i Virgili, Facultat d' Infermeria). Recuperado de http://acise.cat/wp-content/uploads/2017/03/treball-de-fi-de-grau-1.pdf. 
Organización Mundial de la salud (2016). ¿Qué es la promoción de la salud? Recuperado de http://www.who.int/features/qa/health-promotion/es/.

Ossama Nasser Laaoula, A. (2011). La Enfermería Escolar: una necesidad sentida. (Trabajo Fin de Master, Almería, Universidad de Almería. Escuela de Ciencias de Salud). Recuperado de http://docplayer.es/7653848-La-enfermeria-escolar-unanecesidad-sentida.html.

Real Decreto 1231/2001, de 8 de noviembre, por el que se aprueban los Estatutos generales de la Organización Colegial de Enfermería de España, del Consejo General y de Ordenación de la actividad profesional de enfermería. BOE n² 269, de 9 noviembre de 2001. 